\title{
A Production-State Based Approach for Energy Flow Simulation in Manufacturing Systems
}

\author{
Marco Taisch $^{1}$, Bojan Stahl ${ }^{1}$, Federica Vaccari ${ }^{1}$, and Andrea Cataldo ${ }^{2}$ \\ ${ }^{1}$ Politecnico di Milano, Department of Management, Economics and Industrial Engineering, \\ Milan, Italy \\ \{marco.taisch, bojan.stahl\} apolimi.it, \\ \{federica.vaccari\}@mail.polimi.it \\ ${ }^{2}$ Institute of Industrial Technology and Automation National Research Council, Milan, Italy \\ \{andrea.cataldo\}@itia.it
}

\begin{abstract}
Energy Efficiency plays a major role in manufacturing being one of the largest consumers and offering opportunities for cost-savings and improvements. Simulation is an established tool for optimizing manufacturing systems. The paper shows a new production-state based approach for integrating material flow and energy consumption in commercial discrete-event simulation software. Besides typical investigation of production assets, also technical building services as one potential major source for energy consumption are taken into account. The approach considers TBS as energy demand requirements from assets and does not require modeling the behavior of TBS systems. Hence, robust simulation results can be achieved by much faster modeling time.
\end{abstract}

Keywords: eco-factory, energy efficiency, sustainable manufacturing, simulation.

\section{Introduction}

Energy efficiency in manufacturing has raised the attention of research and industry for quite a while. With a consumption of primary energy sources of ca. $30 \%$ and $36 \%$ of $\mathrm{CO} 2$ emission, the manufacturing sector is a promising area for reduction and optimization potential [1]. Gains in energy efficiency have been tackled from various directions including process and technology optimization, identification of barriers and support of drivers, development of adequate performance measures, and development of supporting tools and methodologies.

Especially quantitative and qualitative tools and methodologies can support decision-makers and stakeholders in gathering information, enlarging perspectives, providing ideas and solutions for improving energy efficiency in production systems. Simulation has been identified as a promising tool to address energy efficiency investigations appropriately in research as well industry. 


\section{State of Research}

A remarkable progress has been done in the modeling and simulation of energy efficiency related aspects in manufacturing in the past 5 years. Simulation and modeling is seen as a core information and communication technology in manufacturing towards the next decade [2]. The usefulness of Discrete-Event-Simulation (DES) in manufacturing application is nowadays undisputable and has been demonstrated in various studies [3], hence diverse commercial simulators like Arena, FlexSim, PlantSimulation, Anylogic, just to name a few, support the planning and optimization of manufacturing systems. But the implementation of energy related functions or indicators or other environmental-oriented functions within commercial simulators is not realized yet, although recent empirical studies have shown that companies see the combination of simulation enhanced by the empirical perspective as a supporting methodology [4] and requirements for future eco-oriented factories claim the same issue [5].

Approaches for including energy flows into material flow simulation are merely dependent on two issues: the first one is programming and coding work if the software allows it dependent on the modeling approach chosen, the second one is the scope of the simulation model. Standard simulation tools concentrate on production asset objects like machines, conveyors, robots, handling systems etc. Since the total energy consumption of a production system is not only dependent on production assets but also periphery systems like compressed air and steam generation as well as central technical building services (TBS) such as HVAC, the scope of the model from production assets via periphery systems to central TBS can have a significant impact on the simulation results. A detailed overview and comparison of existing simulation approaches taking into account energy flows is presented in [6].

Using the PlantSimulation software, Schulz and Jungnickel contribute by combining two different approaches [7]: the calculation of energy consumption associated to each manufacturing state of the production system assets starting from the power profile as proposed by Kulus et al. [8] and the calculation of energy consumption associated to the power profile of each process step as proposed by Putz et al. [9]. The approach is very detailed and gives the user a very good estimation of the real system performance. However, in order to apply the simulation tool and to build the model, detailed information in form of power profiles are required to feed the model. Usually that kind of information is difficult to get since it requires additional measurement activity directly at the production asset of the different energy carriers like electricity and air.

The aim of this paper is to present an approach which suggests the inclusion of periphery systems in the modeling without the reproduction of the TBS behavior but consideration as energy demand requirements from the assets. This approach is supposed to deliver robust and sufficient results with much faster modeling time.

\section{Concept Development}

Integrating energy consideration in DES requires enlarging the system of investigation in order to get a comprehensive view of the energy consumption of a production 
system. In a production system energy consumers can be divided twofold: consumers which are directly and indirectly involved in the production. Direct consumers are referred to as production assets. Production assets are machines, robots, working stations, conveyors, transport systems, storages, buffers etc. They are comprised by all elements which foster the material flow and manufacturing processes directly. Indirect consumers are referred to as technical building services (TBS). TBS are units which enable the production conditions, either by servicing production assets with the right form and quantity of energy or by keeping the production environment in the required condition. The first one is referred to as periphery system, whereas the second one is referred to as central TBS. Since the periphery system are connected to the production assets, the following model for DES will consider both production assets and periphery systems in the modeling approach.

The challenge is to integrate periphery systems, which are typical continuous systems, in a discrete event environment. Within the current state of the art, two approaches have been chosen to integrate periphery systems into DES simulation: the first approach is connecting two different software environments, i.e. a discrete-eventsimulator with specialized software for TBS design. This approach tries to reach the highest level of detail and highest granularity of the model built, however large challenges occur in combining the two different models and programming a suitable interface. Another approach is to model both production assets and periphery systems in one software environment. While this approach has been proved to be feasible and applicable, it puts some rigid constraints on the application. First, the simulation environment has to be able to cope with discrete and continuous flows simultaneously. Most commercial and in industry used software do not support this requirement. Second, both production asset and periphery system behavior needs to be modeled in detail, which puts high demands on modeler skills. Third, the efficiency of the approach concerning requested information and output from the simulation compared to modeling effort can be questioned. The intention of this paper is based on previous work in this field, to derive an approach which enables a fast integration of periphery system into discrete event simulation while maintaining short modeling time, easy application in industry and foremost robust results.

The production assets pass through different productive states during their operation cycle. The power requirements of each state are satisfied by different energy carriers and can be constant or not, according to the type of process and part to be worked. The production assets modelled in this work are exemplarily machines, conveyors and robots, to which a specific power profile can be associated. Each profile results from the approximation to the closest polynomial function for each state applying the EnergyBlocks methodology [10]. The integral of the approximated power profile $[\mathrm{kW}]$ calculated along the state interval given by the simulation, provides the calculations of the energy consumptions [kWh] for each entity.

The machine behaviour has been modelled through their productive states. The considered states for the machine are: off, idle -divided in blocking and starvationworking, set-up and failure. From the energy point of view, there exist also the states ramp-up and ramp-down, which are not productive states but which can imply peaks in the power profile and may have an impact on the energy consumption of a 
machine. The energy consumption associated to the peaks has been considered adding the correspondent value to the energy consumptions calculations coming from the simulation. Assuming that the interval and the extension of the peak are known and that the peak cannot be interrupted by other events, the energy calculation is done independently from the simulation environment. Instead of being calculated gaining from the simulation the real duration associated to the peak, the peak energy consumption value is calculated multiplying $3 / 4$ the peak extension for the known peak duration. As a matter of fact, for the reason that the peaks present a cusp shape and that calculating the peak energy consumption as the product of the peak extension for the duration would overestimate it, $3 / 4$ the peak extension has been used as a realistic estimation of the area included under the peak function.

The model has not differentiated where the energy requirements satisfaction of the production assets come from; they could be due to direct electricity feeding or they could come from the periphery system provision through the energy carriers. For each entity the energy consumption calculation is performed considering the equivalent electrical energy consumption for each of the different carrier feeding the equipment. For a machine which receives in input electricity and compressed air, the energy consumption calculation will be performed calculating the sum of the electrical energy consumption and the equivalent electrical energy consumption consumed by the periphery system which feed the machine with compressed air.

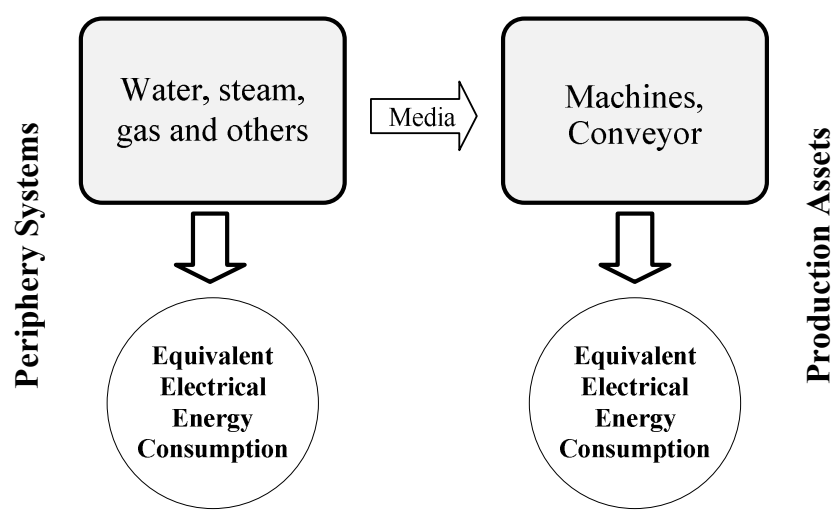

Fig. 1. Production assets and periphery systems energy requirements

The periphery system chosen to be integrated in the model was the compressed air system. It is called by the production assets in specific states -the working and the setup- which need the power coming from the compressed air system for the pneumatic components. The energy consumption associated to the compressed air system has three different consumption ways: the energy provided by the periphery system to the production asset, necessary to satisfy the power requirements of the pneumatic components; the energy required to bring the compressed air system at operational level, filling tanks and pipes; the energy associated to the losses in the compressor and inside the system itself. 
The modelling of the compressed air requirements of the production assets both in terms of power and of associated compressed air has been based on thermodynamic laws. Starting from the calculation of the mechanical work to compress a litre of air passing to the electrical energy consumed by the compressor and arriving then to the calculation of the amount of air required by each single production asset as the equations below show.

$$
\begin{gathered}
W=C_{v} * T *\left(1-\left(\frac{p_{2}}{p_{1}}\right)^{\frac{\gamma-1}{\gamma}}\right) * M_{m} \\
E=\frac{W}{\eta} \\
V_{1}=V_{2} *\left(\frac{p_{2}}{p_{1}}\right)^{\frac{1}{\gamma}}
\end{gathered}
$$

The energy consumption has been then calculated multiplying the energy required to compress one litre of air with the compressed air demand of production assets. Assuming uniform distributed compressed air consumption and energy consumption, dividing them for the value of the processing time for the specific asset and product, it has been possible then to calculate the air consumption per time unit and the power requirements, data required in order to calculate the real time consumption of air and of energy inside the simulation model. The real time calculations inside the model have been needed by the fact that the failures are stochastic events which affect the duration of other states which cannot be considered fixed; furthermore different products can require different power levels and different processing times, making clear why real time calculations are necessary.

The deployment phase has been represented by the translation of the conceptual models in the discrete event simulation software Tecnomatix PlantSimulation. The model performed the calculations of the energy consumed by the production assets real time at three different levels: the production state level, the production assets level and the manufacturing system level. These energy consumption calculations have considered also the energy consumptions coming from the compressed air system as described above.

\section{Application}

The model developed has been assessed in a case study represented by an automated line for the filling of sacks with powders. The automated manufacturing system is composed by two parallel lines, a line for the processing of three types of sacks and a line for the processing of the pallet.

The entities of the system have been customized with the data related to the processing/set-up times, the availability, the speed and the length for the conveyors, the electrical power, the power coming from the compressed air system and the associated consumption of air. Three different tests have been performed on the line used 
as a case study in order to validate the model and to see its application. The different tests have been performed using KPIs articulated at different levels of analysis and dealing with different dimensions - production, economic and energy.

The first test has dealt with the assessment of different parts sequencing: random, big batches and small batches. As it could be easily predictable, the big batches solution has represented the best trade-off of production and energy performances, a result which represented also a validation for the model developed. The simulations have been conducted over a total production of 90 parts, divided in the three tests as the table shows.

Table 1. Part distribution

\begin{tabular}{|c|c|c|c|}
\hline Strategy & Part Type 1 & Part Type 2 & Part Type 3 \\
\hline Random & $17 \%$ & $33 \%$ & $50 \%$ \\
\hline Big batches & 15 & 30 & 45 \\
\hline Small batches & 5 & 10 & 15 \\
\hline
\end{tabular}

While the manufacturing performances remained constant, the energy ones have been shown to change: having big batches represents the best solution in terms of total energy consumption.

Table 2. Consumption and emissions based on batch sizes

\begin{tabular}{|c|c|c|}
\hline Strategy & $\begin{array}{c}\text { Total Energy } \\
\text { Consumption }[\mathrm{kWh}]\end{array}$ & $\mathrm{CO}_{2}$ Emissions $[\mathrm{Kg}]$ \\
\hline Random & 4,005 & 2,127 \\
\hline Big batches & 3,675 & 1,951 \\
\hline Small batches & 3,692 & 1,961 \\
\hline
\end{tabular}

The big batches solution represents the most valuable solution also from the point of view of the energy efficiency performances, meant in terms of energy efficiency (process output/energy input), SEC (1/energy efficiency) and eco-efficiency (parts produced/environmental influence).

The second test conducted dealt with the assessment of the reduction of the processing time for the bottleneck. The reduction of the processing times even implying more energy consumed, assures the best trade-off with the parts produced. The performances have been assessed changing the processing times of the bottleneck, respectively decreasing it by $10 \%$ and $20 \%$.

Table 3. Altering process times

\begin{tabular}{|c|c|c|c|}
\hline Processing Time & $\begin{array}{c}\text { Processing Time } \\
(P 1)[s]\end{array}$ & $\begin{array}{c}\text { Processing Time } \\
(P 2)[s]\end{array}$ & $\begin{array}{c}\text { Processing Time } \\
(P 3)[s]\end{array}$ \\
\hline AS-IS & 4,00 & 8,00 & 9,60 \\
\hline$-10 \%$ & 3,60 & 7,20 & 8,64 \\
\hline$-20 \%$ & 3,20 & 6,40 & 7,68 \\
\hline
\end{tabular}


In this scenario the happening of the failures cannot be forecasted, the outputs are not deterministic and are represented by the average values. The decrease in the processing time of the bottleneck increased the flow rate and decreased the throughput time of the entire manufacturing system. Nevertheless, it did not cause significant changes in the total energy consumption of the manufacturing system, decreasing instead the energy consumption of the bottleneck. The following table shows the global results in terms of manufacturing and energy consumption.

Table 4. Consumptions for altering process times

\begin{tabular}{|c|c|c|c|}
\hline Processing Time & $\begin{array}{c}\text { Flow Rate } \\
\text { [pallet /month] }\end{array}$ & $\begin{array}{c}\text { Total Energy } \\
\text { Consumption } \\
{[\mathrm{kWh}]}\end{array}$ & $\begin{array}{c}\text { Total Air } \\
\text { Consumption [l] }\end{array}$ \\
\hline AS-IS & 18619 & 13991,665 & 4242139,590 \\
\hline$-10 \%$ & 18610 & 13967,169 & 4073167,231 \\
\hline$-20 \%$ & 18702 & 13990,639 & 3925761,366 \\
\hline
\end{tabular}

Finally, the third test has dealt with the doubling of the bottleneck station, bringing to the conclusion that a slightly improvement of the productive performances lead to a worsening of the energy ones, as the table below, reporting the energy efficiency performances, shows.

Table 5. Results for doubling bottleneck

\begin{tabular}{|c|c|c|c|}
\hline & Energy efficiency & SEC & Eco-efficiency \\
\hline AS-IS & 1,331 & 0,751 & 2,506 \\
\hline TO-BE & 1,324 & 0,755 & 2,493 \\
\hline
\end{tabular}

\section{$5 \quad$ Summary and Outlook}

The aim of the paper is to highlight the opportunity of using discrete-event simulation with material flow simulation as a standard tool in manufacturing and enrich it with taking into consideration energy consumption. The approach is based on productionstates of the production assets and has shown that implementing energy measures is possible and can lead to a widened decision sense. The approach has shown the usefulness of the equivalent energy consumption, which allows also the detailed planning, sizing, and consideration of periphery systems within material flow simulation without modelling the detailed behaviour of the TBS but including it in the energy demand requirements of the assets. The enlarged provision of information for the user from the environmental perspective point of view might be an opportunity for considering green issues in production system optimization.

Acknowledgements. The results were developed within the research project EMC2 Factory (Eco Manufactured transportation means from Clean and Competitive Factory) funded by the European Commission within FP7 (285363). 


\section{References}

1. Interntional Energy Agency, Worldwide Trends in Energy Use and Efficiency, Key Insights from IEA Indicator Analysis (2008),

http: / / www. iea.org/publications/freepublications / publication/Indicators_2008-1.pdf

2. Taisch, M., Stahl, B., Tavola, G.: ICT in Manufacturing: Trends and Challenges for $2020-$ An European View. In: Proceedings of the IEEE 10th International Conference on industrial Informatics, Beijing, July 25-27, pp. 941-946 (2012)

3. Jahangirian, M., Eldabi, T., Naseer, A., Stergioulas, L.K., Young, T.: Simulation in manufacturing and business: A review. European Journal of Operational Research 203, 1-13 (2010)

4. May, G., Taisch, M., Stahl, B., Sadr, V.: Toward Energy Efficient Manufacturing: A Study on Practices and Viewpoint of the Industry. In: Emmanouilidis, C., Taisch, M., Kiritsis, D. (eds.) Competitive Manufacturing for Innovative Products and Services (2012)

5. Taisch, M., Stahl, B.: Requirements analysis and definition for eco-factories: the case of EMC2. In: Emmanouilidis, C., Taisch, M., Kiritsis, D. (eds.) Competitive Manufacturing for Innovative Products and Services (2012)

6. Thiede, S.: Energy efficiency in manufacturing systems. Dissertation TU Braunschweig (2012)

7. Schulz, S., Jungnickel, F.: A General Approach to Simulating Energy Flow in Production Plants via Plant Simulation. In: Seliger, G., Kilic, S.E. (eds.) Proceedings of the 10th Global Conference of Sustainable Manufacturing, Towards Implementing Sustainability, pp. 724-729 (2012)

8. Kulus, D., Wolff, D., Ungerland, S.: Simulating Energy Consumption in the Automotive Industry. Use Cases of Discrete Event Simulation. Springer, Berlin (2012)

9. Putz, M., Schlegel, A., Lorenz, S., Schulz, S., Franz, E.: Gekoppelte Simulation von Material- und Energieflüssen in der Automobilfertigung. In: 14. Tage des Betriebs- und Systemingenieurs, Chemnitz, pp. 134-145 (2011)

10. Weinert, N., Chiotellis, S., Seliger, G.: Methodology for planning and operating energyefficient production systems. CIRP Annals - Manufacturing Technology 60, 41-44 (2011) 